\title{
Difference in operation of Mouse and Touchscreen in older adults
}

\author{
Anika Steinert ${ }^{1}$, Marten Haesner ${ }^{1}$, Julie L. O Sullivan ${ }^{1}$, Elisabeth Steinhagen- \\ Thiessen $^{1}$ \\ ${ }^{1}$ Geriatrics Research Group, Charité - Universitätsmedizin Berlin \\ \{anika.steinert, marten.haesner, julie.osullivan, elisabeth.steinhagen-thiessen\}@charite.
}

\begin{abstract}
Within the present study, an investigation with 50 older adults aged over 60 years was conducted. One aim of the study was to identify differences in usage of an internet platform for cognitive training with two different input devices. For this purpose, the subjects had to solve tasks on both PC and tablet computers. The success rate and the amount of time required to solve the tasks were recorded in a standardized manner. Additionally, participants were asked about the subjective advantages of both terminal devices and about their general preference. Overall, we found hardly significant differences in success rates and task-solving time. Contrary to other studies, where participants had to choose a defined target or perform a short specific task the older adults in the present study made nearly the same number of mistakes and needed almost the same time for solving the assigned tasks when using the $P C$ and the tablet.
\end{abstract}

Keywords: input device, tablet, computer usage, seniors, human-computer-interaction

\section{Introduction}

A certain degree of computer literacy as well as the ability to access and use the internet are essential in order to fully participate in today's society [1]. Especially older people could benefit from internet access in a social and psychological way [2,3]. There are several studies pointing out that the use of the internet can reduce social isolation and facilitate communication with family and friends [4]. There is a growing interest in the use of internet games as a means to educate and train people [5]. The combination of game technology and therapeutic methods can lead to beneficial therapeutic effects [6]. Especially people with disabilities or age-related functional decline can benefit from new developed Online Cognitive Training Platforms. In recent years, many clinical trials were conducted giving evidence that serious cognitive games have a significant positive impact on older person's mental and physical health and wellbeing [7]. Moreover, the motivational approach can help older people exercising regularly in their home environment.

Although older adults will mostly benefit from online cognitive training platforms, only a small number of this age group are using them. A reason might be lacking usability of online games and problems with the operation of new technical devices.

In general, standard computers and laptops are mainly operated with a mouse, while mobile devices such as tablets or smartphones are normally accessed directly by touching the screen with a finger. Devices operated by touchscreen are ubiquitous and their development marks an important step in the on-going technological revolution. In addition to public information displays in banks, airports or railway stations, mobile multimedia devices such as smartphones or tablets are operated via touchscreen. In comparison to the mouse, directly touching elements on a tablet is seen as a more "natural" alternative [8].

Due to the demographic development, an increasing amount of older people need to become acquainted with this new way of interacting with a computer. However, touchscreens were developed in the last 20 years, thus many older adults find them difficult to use.

Operating a device via touchscreen could provide a possibility to overcome some of the problems older adults have with computer usage. For example, using a computer mouse generally requires a high degree of fine motor skills. Hence, reduced motor skills - as are common among many older people - can lead to unnecessary barriers in computer usage. In contrast, touchscreens enable a more 
direct input an require less fine motor skills than regular computer mice [9]. However, both the direct and the indirect control offer advantages and disadvantages. The mouse control allows greater precision and there is a higher number of controls (left/right button, scroll wheel) as opposed to a single finger when operating a touchscreen. Furthermore mouse control allows accelerated movements (ability to have a non-linear relationship between the speed of moving the pointing device and the on-screen pointer: moving the mouse fast makes the on-screen pointer move even faster) and offers a visible cursor. On the other hand, touch operations also show some clear benefits compared with the use of a PC mouse. When moving the hand from one input device such as the keyboard to another, there is no time required which make touchscreens suitable for mobile use [9]. Various studies have shown that the handling of touchscreens is easier to learn - people can easily learn how to use them, even without prior experiences or instructions [10], and they seem more fun to use [11]. However, touchscreen operation does not seem suited for executing complex tasks or writing long messages or texts.

In the present study, we will investigate how older adults perform with a specific serious gaming platform for cognitive training. The authors wanted to investigate the differences when using the platform on a PC and a tablet. It is also interesting which input device were preferred by older adults who used both a tablet and a PC for solving several tasks. Following the results of Robert et al. [12] regarding recommendations for the use of serious games in people with alzheimer's disease the authors want to derive generally recommendations from the results for developers of serious gaming platforms.

\section{Methods}

\subsection{Participants}

A total of 50 participants took part in the study. On average, the subjects were aged approximately 71 years (Range: 61 -93 years) and it was a mostly well-educated (over $50 \%$ had a university degree) and urban sample (from the city of Berlin). The majority of the participants reported frequent use of both the computer and the internet. In contrast, tablets, smartphones or other touchscreen devices were only used by a small part of the older adults in this sample. Only $22.4 \%$ of the participants had used a tablet prior to this study. Overall, the sample is highly interested in technology. Two thirds of the participants reported an interest in technology and technical issues. In a section of a validated technology commitment questionnaire [12] the participants achieved an average score of 15 points (20 points being the maximum score).

\subsection{Apparatus}

In the study we investigated the performance of older adults with an online platform on a computer (Screen size: 22 inch) and a tablet (10 inch). The platform was developed within the German, publicly funded research project LeVer. The aim of the project was to create an innovative online service that can help to prevent and rehabilitate cognitive impairments in elderly people. The platform consists of four areas:

- The cognitive training

- A personal area, with the possibility to communicate with other users by messaging or audio-video communication

- An information area, where you can find specific information on healthy aging, nutrition and cognition and a forum

- Settings, for adjusting the volume or the type size

The PC and tablet version of the platform were identical regarding structure, design and functions, there only difference was the size of presentation due to different screen sizes.

\subsection{Design}

After an initial screening, participants completed several questions regarding their sociodemographic background, computer and tablet usage and self-efficacy. The order in which devices were presented and tested by the participants was randomized. After randomization the participants were asked to perform six tasks on tablet and PC: 
- $\quad$ Log in on the platform (Figure 1)

- Write a comment

- $\quad$ Return to main page

- Write a message (Figure 2)

- $\quad$ Adjust a setting

- $\quad$ Start an Audio-Video Call

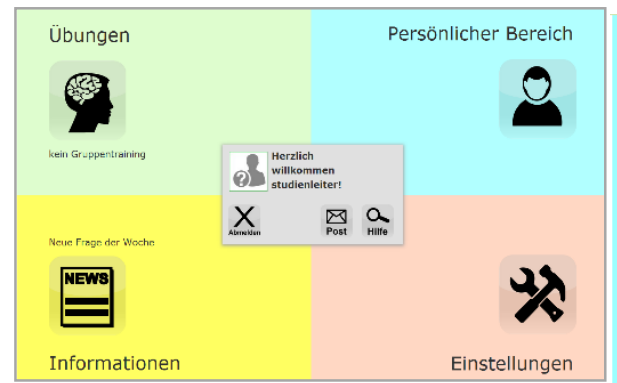

Figure 1: Main page

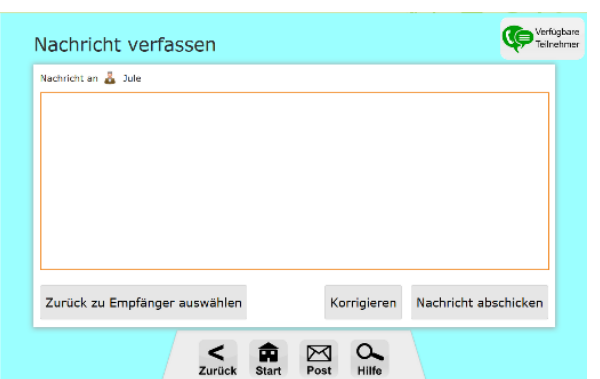

Figure 2: Sending a message

Before starting with the first task, the participants were briefly instructed about the most important functions of the platform and about the use of a tablet keyboard. Subsequently, the participants were required to solve the six tasks using a mouse and a touchscreen, presented in counterbalanced order. In several previous studies, touchscreens were rated as the most "natural" or "compelling" input devices $[8,10]$. Due to the fact that "naturalness" is not an objective performance measure, speed, performance, error rates and user preference when using a touchscreen or mouse were analyzed for each task. Errors were protocolled in a standardized manner.

\begin{tabular}{|c|}
\hline Screening \\
age, cognition, fine motoric, hearing and vision \\
Assesments \\
CERAD, paper folding test, tower of Hanoi \\
Questionnaires \\
कociodemographic data, self-effiacy, technology commitment \\
\hline First run with PC or tablet \\
six tasks \\
Questionnaires \\
\hline usability, design, user friendliness \\
Second run with PC or tablet \\
six tasks \\
Questionnaires \\
\hline prefferred device, advantages and disadvantages of PC and tablet \\
\hline
\end{tabular}

Figure 3: Methodology of the study

After the participants conducted the tasks for the first time with the first device, they were asked about usability (AttrackDiff Questionnaire, [13]; System Usability Scale, [11]; Rating Scale of Mental Effort (RSME), [15]), design and user-friendliness of the platform. After the second session, they were asked about their preferred device and the advantages and disadvantages of PC and tablet $\mathrm{PC}$ respectively (figure 3 ).

\section{Results}


The participants had to solve six tasks on the platform on both devices - PC and tablet. The tasks included typical functions of a webpage such as login or writing messages. In the present study, performance regarding the time required and the mistakes made, when using the same platform on PC and tablet, were investigated. For each task, the participants could achieve a maximum of two points - if they managed to solve the task without help and without making a mistake. Without making a mistake meant in this case finding the right solution with the first attemp. Furthermore we assessed which device was preferred by older adults when using the platform.

\subsection{Duration and success rate of single tasks}

Task 1: $\log$ in

The first task was to login to the platform, similar to other online platforms. The most frequent error when using the PC was writing the name or the password incorrectly $(n=3)$. On the tablet, the most frequent error was closing the keyboard after text input $(n=7)$. The virtual keyboard automatically opens and closes by touching the empty entry fields. Apparently, this procedure was not intuitive for many participants, as most participants spent a long time searching for the keyboard and tried touching several different buttons. The participants needed significantly less time conducting the tasks on the PC in comparison to the tablet $(41 \mathrm{~s} / 76 \mathrm{~s}, \mathrm{~T}=-2.573, \mathrm{p}=.013)$. In this task $66.7 \%$ of the subjects using the PC and $48 \%$ of the tablet users were successful (n.s.).

\section{Task 2: Write a comment}

The second task was to comment on the discussion topic in the forum: "How do you remember important dates?" The major challenge for this operation on PC was finding the correct menu option in the three-level hierarchical system $(n=9)$. On the tablet, many participants made mistakes when open and closing the keyboard after entering a comment (each $n=6$ ), and similar to PC choosing the right area on the main page proved difficult $(n=6)$. Due to the fact that the participants were allowed write any comment irrespective of length, the time was not measured during typing. Nevertheless, the participants needed more time for this task on the tablet than on the PC. However, the difference was not statistically significant $(95 \mathrm{~s} / 78 \mathrm{~s}, \mathrm{n} . \mathrm{s}$.). The success rate showed no essential differences between operation on PC and tablet ( $28 \% / 33.3 \%$, n.s.). Generally, this task led to major problems among the participants; therefore the lowest success rate of all tasks was achieved on this task.

Task 3: Return to the main page

Afterwards, the users had to return to the homepage of the platform. There were three ways of doing this: By choosing the return button or the "Home" symbol from the platform's navigation bar, or the tablet's own return button which could be used to return to the previous webpage. The main problem was that the participants did not recognize the "Home" symbol which depicted a house, as a link to the main page, hence they often use the return button. There were no significant differences between the users who used the PC and the Tablet users regarding the duration for the task $(10 \mathrm{~s} / 12 \mathrm{~s}$, n.s.). There were also no statistical significantly differences in the success rate - on PC $70.8 \%$ of the participants were successful and on tablet only $48 \%$ succeeded.

Task 4: Write a message

The fourth task for the participants was to write a message on the platform. Similar to the second task the time was not measured during text input. Both on PC and tablet, the main problem was that the participants chose a wrong area on the main page $(n=10, n=9)$. Some of the tablet users had problems with closing the keyboard to send the message $(n=7)$. Even in this task the participants who used the PC needed significantly less time than the seniors who used the tablet $(56 \mathrm{~s} / 75 \mathrm{~s}$, $\mathrm{p}=.018$ ). However, there were only few differences in success rate $-50 \%$ of the PC users and $40 \%$ of the tablet users succeeded.

Task 5: Adjust a setting

In the fifth task, participants were asked to open the "settings" menu and choose the option that they would like to be informed via email about news or changes on the platform. In contrast to the previous tasks, the tablet group performed significantly better on this task (56\%) compared to the group who used the PC $\left(12.5 \%, \chi^{2}=10.23, \mathrm{p}=.001\right)$. Furthermore, the participants using the tablet did not needed significantly less time for solving the task than those using the PC (61 s/ 74 s, n.s.).

Task 6: Start an audio-video call 
The last task for the participants was to start an AV call. The most frequent mistake both for the PC and tablet users was that the participants were not able to choose a contact to start the call $(n=18$, $\mathrm{n}=17)$. The success rate of the seniors using the tablet was higher $(36 \%)$ than the success rate of the PC users (29.2 \%, n.s.). However, hardly any differences were found regarding the duration of solving the task between the PC users ( $57 \mathrm{~s})$ and the tablet users (56 s, n.s.; table 1).

Table 1: Duration and success rate Task 1-6

\begin{tabular}{c|c|c|c|c}
\hline & \multicolumn{2}{|c|}{ Duration (in s) } & \multicolumn{2}{c}{ Success (in \%) } \\
\hline Task & PC & Tablet & PC & Tablet \\
\hline 1 & 41 & 76 & 66.7 & 48.0 \\
\hline 2 & 78 & 95 & 33.3 & 28.0 \\
\hline 3 & 12 & 10 & 70.8 & 48.0 \\
\hline 4 & 56 & 75 & 50.0 & 40.0 \\
\hline 5 & 74 & 61 & 12.5 & 56.0 \\
\hline 6 & 57 & 56 & 29.2 & 36.0 \\
\hline & 53 & 62 & 43,8 & 42,7 \\
\hline
\end{tabular}

\subsection{Preferred terminal of older adults}

In a short questionnaire, the participants were asked about their preferred input device. For a longterm operation of the platform, $83.7 \%$ of the older adults rated the PC as more suitable. For nearly $70 \%$ of the participants, the use of the PC was easier than operating the platform with a tablet. Accordingly, two thirds would prefer to use the PC at home when handling the platform (figure 4). The graphical interface was rated as mostly equivalent on both devices. In contrast, the PC received better ratings regarding suitability and simplicity whereas almost half of the participants believed that the general operation of a tablet would be easier to learn (figure 4).

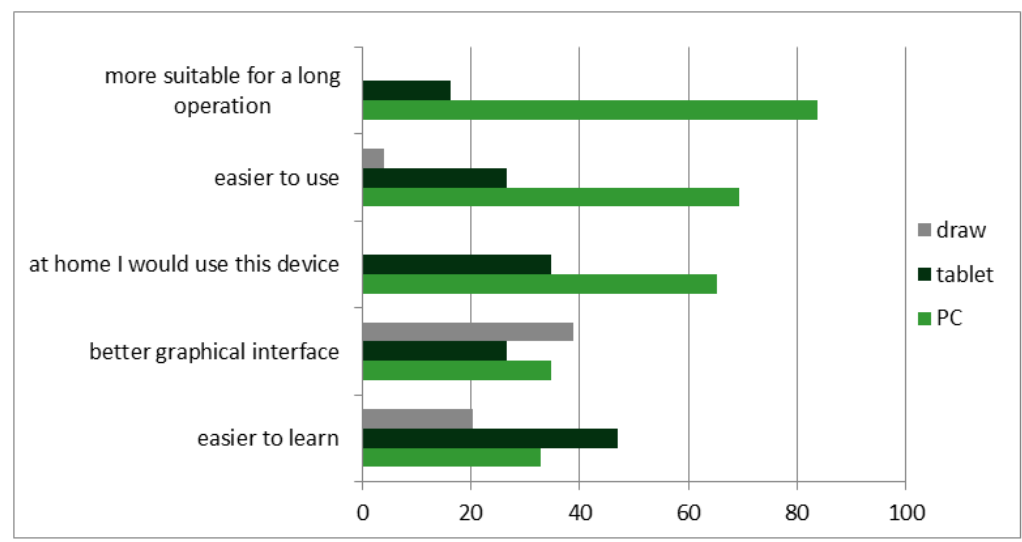

Figure 4: Preference Tablet PC, \%

Furthermore, the participants were asked about the subjective advantages of both devices (table 2). The main advantage of the tablet was seen in the portability of the gadget. The main advantage of the PC was the greater size of the screen in contrast to the tablet.

Table 2: Advantages of PC and tablet

\begin{tabular}{l|c|l|c}
\hline Advantages Tablet* & Frequency (\%) & Advantages PC* & Frequency (\%) \\
\hline Portability & 88 & large screen & 50 \\
\hline small/ handy & 50 & more comfortable & 40 \\
\hline easy to use & 14 & already accustomed & 28 \\
\hline no mouse or keyboard required & 8 & more options & 24 \\
\hline possibility to take and show pictures & 4 & larger memory space & 8 \\
\hline
\end{tabular}

* Multiple answers possible 


\section{Limitations}

In the present study we investigated a small homogenous sample, which included primarily well educated and study-experienced older adults. Because of the homogeneity of the sample, it is possible that our findings will not apply to samples that are more heterogeneous. Furthermore, a generalization of the findings is not possible because of the use of the specific LeVer Platform.

\section{Conclusion}

Although there has been some research investigating the differences between several input devices, the present study has found new results for the elderly in this field of research. In contrast to previous studies, which showed significant differences between the mouse and touchscreen operation, there were only small differences between the input devices in the present study. Neither research better performance with mouse interaction $[16,17]$ nor studies claiming that touchscreens are more suitable for input can be confirmed with this study. There were only marginal differences in performance speed and success rate between PC and tablet. In the first run, tasks requiring text input could be solved faster and with more success on the PC. However in the second run, the gap between performance in mouse and touchscreen was reduced. After the second run, participants showed no performance differences between tablet and PC. This result seems quite remarkable, considering that the majority of participants reported frequent use of a computer but had little or no experience with touchscreen devices. After a short introduction and a brief practice run, the older adults were able to operate the touchscreen device. This finding may have been influenced by the high technology interest and technology competence within our sample. Similar to the experiments of Meyer et al. [16] and Sears and Shneiderman [17] the subjects preferred the mouse as an input device. This may have been due to the high computer experience of the majority of the participants. In concurrence with findings of Holzinger [10], who reported that the touchscreen is the most natural of all input devices and even children can easily learn how to use it, the participants of the present study also reported that touchscreen operation was easier to learn than mouse use. With an extensive teaching concept both input devices can be learned by older adults. Due to the significantly decreased number of errors in the second run, we can assume improved performance with appropriate training. From the results, there are requirements for UI designers and future work. Especially an improvement of the keyboard must be focused when designing online platforms on a touchscreen device for older adults. The results of Robert et al. [18] already show, that a large number of steps overwhelm older adults with Alzheimer disease, and lower the benefits of a game. Utilizing less steps and creating a flat web site hierarchy with fewer subpages can be essential in developing senior-friendly web pages to avoid navigation mistakes. In addition, a senior friendly manual and a support system must be prepared for the older adults when conducting a study as well as when leaving older adults alone with the devices. To ensure older adults can handle a webpage for serious gaming they should train independently.

To validate these results, future studies with a longer period of investigation with a larger sample in more diverse populations should be conducted. Long term studies should also be conducted to investigate long term usage of the devices.

Key points:

- The study shows that the time required for tasks and the success rate are not significantly constrained by the input device

- Training and technical assistance seem to be essential for both tablet and PC usage

- Opening and closing the keyboard on a touchscreen device must be more intuitive

- There are differences in types of errors - with the tablet the participants made more mistakes in tasks which required text input

- There is a need for more research in differences between several input devices with a larger sample and a longer use of the devices

- Motoric and sensoric abilities of the target group must be focused when designing a serious gaming platform for older adults 


\section{Acknowledgement}

This article was produced as part of a project which was supported by the German Federal Ministry of Education and Research under grant number 16SV5638K. Responsibility for the contents of this publication lies with the authors. In conclusion, we would also like to thank our partners German Research Center for Artificial Intelligence, Phoenix Software GmbH, Vitapublic GmbH and Evangelisches Geriatriezentrum.

\section{References}

[1] Koopman-Boyden PG, Reid SL. Internet/e-mail usage and well-being among 65-84 year olds in New Zealand: Policy implications. Educ Gerontol. 2009;35(11):990-1007. http://dx.doi.org/10.1080/03601270902917745

[2] Gerber E. The Benefits of and Barriers to Computer Use for Individuals Who Are Visually Impaired. J Vis Impair Blind. 2003;97(9):1-28.

[3] Taveira A, Choi S. Review Study of Computer Input Devices and Older Users. Int J Hum Comput Interact. 2009;25(5):455-74. http://dx.doi.org/10.1080/10447310902865040

[4] Segrist KA. Attitudes of Older Adults Towards a Computer Training Program. Educ Gerontol. 2004;30(7):563-71. http://dx.doi.org/10.1080/03601270490466958

[5] Durkin K. Videogames and young people with developmental disorders. Rev Gen Psychol. 2010;14(2):122-40. http://dx.doi.org/10.1037/a0019438

[6] Kato PM. Video games in health care: Closing the gap. Rev Gen Psychol. 2010;14(2):113-21. http://dx.doi.org/10.1037/a0019441

[7] Raessens J, (pub.). Handbook of computer game studies, Cambridge, Mass.: MIT Press; 2011.

[8] Forlines C, Wigdor D, Shen C, Balakrishnan R. Direct-touch vs. Mouse Input for Tabletop Displays. In: Proceedings of the SIGCHI Conference on Human Factors in Computing Systems. New York, NY, USA: ACM; 2007. p. 647-56. http://dx.doi.org/10.1145/1240624.1240726

[9] Nielsen J. Mouse vs. Finger as Input Device. 2012. online: http://www.nngroup.com/articles/mouse-vs-fingers-input-device/

[10] Holzinger A. User-Centered Interface Design for disabled and elderly people: First experiences with designing a patient communication system (PACOSY). In: Computers helping people with special needs. Springer; 2002. p. 33-40. online: http://link.springer.com/chapter/10.1007/3-54045491-8_8

[11] Häikiö J, Wallin A, Isomursu M, Ailisto H, Matinmikko T, Huomo T. Touch-based User Interface for Elderly Users. In: Proc.s 9th Int.1 Conf.on Human Computer Interaction with Mobile Devices and Services. New York, NY, USA: ACM; 2007 [p. 289-96] http://dx.doi.org/10.1145/1377999.1378021

[12] Neyer FJ, Felber J, Gebhardt C. Entwicklung und Validierung einer Kurzskala zur Erfassung von Technikbereitschaft. Diagnostica. 2012;58(2):87-99. http://dx.doi.org/10.1026/00121924/a000067

[13] Hassenzahl M. Hedonic, Emotional, and Experiential Perspectives on Product Quality. In: Ghaoui C, Herausgeber. Encyclopedia of Human Computer Interaction. IGI Global; 2005 [cited 13th May 2013]. S. 266-72. online: http://www.igi-global.com/chapter/hedonic-emotionalexperiential-perspectives-product/13133

[14] Bangor A, Kortum PT, Miller JT. An empirical evaluation of the System Usability Scale. Int J Hum-Comput Interact. 2008;24(6):5774-594. http://dx.doi.org/10.1080/10447310802205776

[15] Zijlstra FR., Doorn L. The Construction of a Scale to Measure Perceived Effort. Technical Report. Delft University of Technology; 1985.

[16] Meyer S, Cohen O, Nilsen E. Device comparisons for goal-directed drawing tasks. In: Conference companion on Human factors in computing systems. ACM; 1994. p. 251-2. http://dx.doi.org/10.1145/259963.260468

[17] Sears A, Shneiderman B. High precision touchscreens: design strategies and comparisons with a mouse. Int J Man-Mach Stud. 1991;34(4):593-613. http://dx.doi.org/10.1016/00207373(91)90037-8

[18] Robert PH, König A., Amieva H., Andrieu S., Bremond F., Bullock R., et. al., Recommendations for the use of Serious Games in people with Alzheimer's Disease, related disorders and frailty. Front Aging Neurosci. 2014;6. doi: http://dx.doi.org/10.3389/fnagi.2014.00054 